\title{
Fast gene disruption in Trichoderma reesei using in vitro assembled Cas9/gRNA complex
}

\author{
Zhenzhen Hao and Xiaoyun Su ${ }^{*}$ (D)
}

\begin{abstract}
Background: CRISPR/Cas9 has wide application potentials in a variety of biological species including Trichoderma reesei, a filamentous fungus workhorse for cellulase production. However, expression of Cas 9 heterologously in the host cell could be time-consuming and sometimes even troublesome.

Results: We tested two gene disruption methods in T. reesei using CRISPR/Cas9 in this study. The intracellularly expressed Cas9 led to unexpected off-target gene disruption in T. reesei QM9414, favoring inserting 9- or 12-bp at 70- and 100-bp downstream of the targeted ura5. An alternative method was, therefore, established by assembling Cas9 and gRNA in vitro, followed by transformation of the ribonucleoprotein complex with a plasmid containing the pyr4 marker gene into T. reesei TU-6. When the gRNA targeting cbh1 was used, eight among the twenty seven transformants were found to lose the ability to express $\mathrm{CBH1}$, indicative of successful cbh1 disruption through genome editing. Large DNA fragments including the co-transformed plasmid, chromosomal genes, or a mixture of these nucleotides, were inserted in the disrupted cbh1 locus.
\end{abstract}

Conclusions: Direct transformation of Cas9/gRNA complex into the cell is a fast means to disrupt a gene in T. reesei and may find wide applications in strain improvement and functional genomics study.

Keywords: CRISPR/Cas9, Trichoderma reesei, Gene disruption, Ribonucleoprotein

\section{Background}

Trichoderma reesei is a well-known filamentous fungus workhorse for production of cellulase and other industrially important proteins [1]. Gene disruption is a critical technique for functional genomics study of $T$. reesei. From a practical perspective, deleting key regulatory genes that repress cellulase expression has proved to be very useful in strain improvement [2]. In addition, removal of major cellulase and protease genes is an essential step towards creating a platform strain for heterologous protein production [3]. Therefore, a method is needed to efficiently disrupt the genes of interest in $T$. reesei.

In $T$. reesei, gene disruption is routinely achieved by gene replacement through homology-based DNA recombination (HDR) [4]. However, one should note that the efficiency of HDR in T. reesei is notoriously low.

\footnotetext{
* Correspondence: suxiaoyun@caas.cn

Key Laboratory for Feed Biotechnology of the Ministry of Agriculture, Feed Research Institute, Chinese Academy of Agricultural Sciences, No. 12 South Zhongguancun Street, Beijing 100081, China
}

Knocking out ku70 or mus53 involved in non-homologous end-joining (NHEJ) can improve the HDR rate [5], but time is needed to obtain a recipient strain with abated NHEJ function. The CRISPR/Cas9 system (with CRISPR standing for clustered regularly interspaced short palindromic repeats) is the bacterial type-II adaptive immune system [6], which can serve as an alternative means for gene disruption in $T$. reesei. In this system, the gRNA recognizes specific target DNA sequence, forms DNA/RNA duplex at the locus of recognition, and guides the endonuclease Cas9 to cleave the DNA. The CRISPR/Cas9 technology has been widely used for genome editing in mammalian cells, plants, and microbes. Genome disruption using CRISPR/Cas9 has been reported for a variety of filamentous fungi [7], which commonly requires expression of cas 9 in vivo. In contrast, transformation of the in vitro assembled Cas9 and gRNA complex provides a fast means of genome disruption [8], which however has not been reported in T. reesei.

(c) The Author(s). 2019 Open Access This article is distributed under the terms of the Creative Commons Attribution 4.0 International License (http://creativecommons.org/licenses/by/4.0/), which permits unrestricted use, distribution, and reproduction in any medium, provided you give appropriate credit to the original author(s) and the source, provide a link to the Creative Commons license, and indicate if changes were made. The Creative Commons Public Domain Dedication waiver (http://creativecommons.org/publicdomain/zero/1.0/) applies to the data made available in this article, unless otherwise stated. 
In the present study, it was first found that the intracellularly expressed Cas9 led to unexpected off-target gene disruption in T. reesei QM9414. An alternative means, i.e. direct transformation of the in vitro assembled Cas9/gRNA, was evaluated for its efficacy in gene disruption using the cellobiohydrolase I (cbh1) gene as a model. The sequences of the disrupted gene were analyzed, identifying insertion of large chromosomal DNAs or plasmid fragments.

\section{Results}

Intracellularly expressed Cas9 led to unexpected offtarget genome editing

The cas 9 gene has been expressed constitutively or induced successfully in $T$. reesei, in both cases which can be used in successful genome editing in QM6a and RUT-C30 [7]. The codon-optimized cas9 gene [7] was synthesized, ligated downstream of the strong, constitutive $p d c 1$ promoter, and transformed into $T$. reesei QM9414. Nine positive transformants were obtained, among which strain $\mathrm{C} 5$ had the highest cas9 transcript level (determined by RT-qPCR, data not shown) and was therefore selected for subsequent gene disruption.

The ura5 gene has been used as the target when the CRISPR/Cas9 technology was established in $T$. reesei [7]. The in vitro transcribed gRNA targeting the same ura5 locus was transformed into C5. Nineteen 5-fluoroorotic acid (5-FOA) resistant transformants were obtained. While no mutation was found in the expected editing locus for all these transformants, seven had an unexpected insertion of 12-bp at the 100-bp downstream of the expected editing locus (Fig. 1, Additional file 1: Figure S1). One (QM9414-C5/T18) had an insertion of 9-bp, while another (QM9414-C5/T13) had a deletion of this 9-bp fragment, at the 70-bp downstream of the expected editing locus. Interestingly, the inserted short DNA fragments were a direct repeat of the nucleotides downstream of the insertion site. Moreover, two direct repeats (7- and 11-bp, respectively) were found in both sites (Fig. 1), suggestive of biased off-target editing.

\section{Designing gRNA for $c b h 1$ gene disruption}

Direct transformation of Cas9/gRNA complex may be an alternative for genome editing. Indeed, this technique has already been successfully used for gene disruption in various filamentous fungi including Mucor circinelloides [9], Aspergillus fumigatus [10], and Fusarium oxysporum [11]. For those filamentous fungi that are difficult to manipulate, the transformantion of Cas9/gRNA complex provides a means to speed up genome editing. With this advantage, nevertheless, there was no report of using such a technique in $T$. reesei. $\mathrm{CBH} 1$ is the major cellulase secreted by $T$. reesei, accounting for above $50-60 \%$ in the fermentation broth [12]. Disruption of $c b h 1$ will result in loss of the major band on SDS-PAGE gel, facilitating identification of the strains with successful genome editing. Therefore, for ease of verification, cbh1 was selected as the target gene. Three different gRNAs on cbh1 were designed and synthesized by in vitro transcription. Cas9 and a certain gRNA was incubated with the BcuI-linearized pT3cbh1 plasmid (4717-bp) containing the cbh1 gene. gRNA-3 was the best one to guide Cas9-mediated digestion of pT3cbh1 into two DNA fragments with expected sizes (Fig. 2).

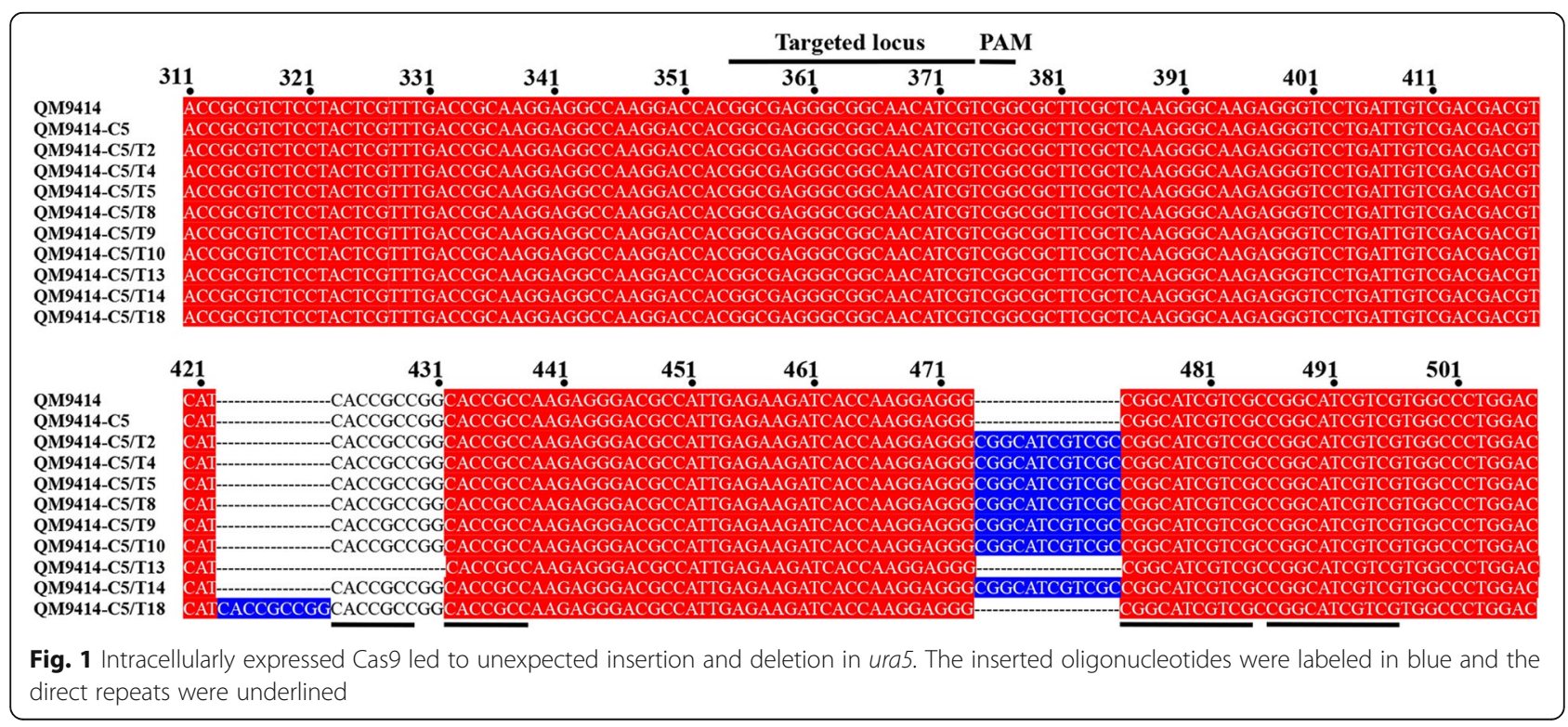




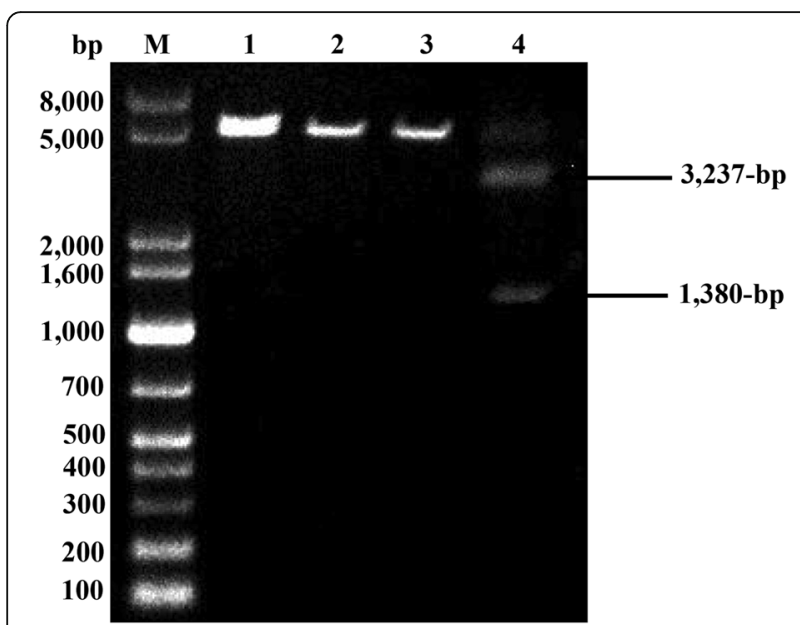

Fig. 2 In vitro digestion of cbh1 by Cas9 and gRNAs. M: DNA molecular mass marker; lane 1: Bcul-linearized plasmid; lane 2-4: Bcul-linearized plasmid incubated with Cas9 and gRNA1 (lane 2), gRNA2 (lane 3), and gRNA3 (lane 4)

\section{Disruption of $c b h 1$ involved insertion of large DNA fragments}

Twenty-seven transformants were obtained in two transformations of Cas9/gRNA with pSKpyr4 in TU-6. Through SDS-PAGE analysis, it was suggested that eight among the 27 transformants could have the cbh1 gene disrupted since the band corresponding to $\mathrm{CBH} 1$ disappeared (Fig. 3A). Interestingly, in these strains, expression of other proteins apparently increased (Fig. 3A), similar to improved secretion of a heterologous lipase in a cbh1-silenced $T$. reesei strain [13]. Using the primers specific for cbh1 (Additional file 1: Table S1), a DNA fragment of 1676-bp was amplified from the parent strain TU-6 (Fig. 3B), as well as from the nineteen transformants still secreting the CBH1 protein (data not shown). Sequencing of all these DNA fragments from these transformants indicated no mutation in the cbhl gene. Using the same PCR condition, no DNA fragment corresponding to the 1676-bp could be amplified from the transformants (except T1) that did not secret CBH1. It has been pointed out that in Nodulisporium sp., transformation of a linear marker plasmid into the cas9-expressing host cell leads to insertion of the marker gene in the gRNA targeting locus [14]. It was hypothesized that similar integration of large DNA fragments could take place. Therefore, the PCR condition was modified, mainly by lengthening the extension time, which would allow amplification of larger DNA fragments. Using this method, we successfully amplified DNA fragments from five transformants (Fig. 3B). The sizes of the DNA fragments from four transformants (T2, T7, T8, and T9) were from 5- to 8-kb, while that for one transformant (T1) was 1.9-kb.
Further sequence analyses indicated that there were gene editing events at the expected $c b h 1$ locus. Deletion of 91 705-bp in cbh1 was observed in T2, T7, and T9 (Fig. 3D). The inserted fragments varied in length and sequence by containing: i) the transformed plasmid (T2, T7, and T8); ii) a chromosome fragment (T1); or iii) mixed chromosome and plasmid sequences (T9, Fig. $3 C \& \mathrm{D})$. Although $\mathrm{T} 2$ and $\mathrm{T} 7 \mathrm{had}$ different deletions, they had the same inserts. It seemed that the pSKpyr4 DNA fragments starting from nucleotide 23 were favored in these gene rearrangement events (Fig. 3D). The underlying reason for this is not known. All five had another fragment ("other insert" in Fig. 3C\&D) which was not from pSKpyr4 or $T$. reesei chromosome but from $E$. coli chromosome (T1) or an unidentified plasmid(s) (T2, T7, T8, and T9), which was likely contaminants from pSKpyr4 preparation.

\section{Discussion}

The intracellularly expressed Cas9 successfully disrupts the ura5 gene in the $T$. reesei QM6a and RUT-C30 strains by creating small deletions [7]. However, disruption of the same gene was quite different in QM9414 in this study since no such small deletions were observed in the 5-FOA-resistant transformants. In contrast, insertion (or less frequently, deletion) of small DNA fragments was found downstream of the genomic locus expected for editing. These mutations resulted in 3-4 amino acids duplication (or 3 amino acids deletion) in the ura5-encoded orotate phosphoribosyl transferase, which might interfere with the normal function of this enzyme, thereby enabling the transfromants to resist the toxicity of 5-FOA. Since the sequence of gRNA was the same as that used in [7], the off-target genome editing was unexpected. In the previous report [7], QM6a and RUT-C30 were used as the cas9-recipient strains. Being cellulase hyperproducer derivatives of QM6a, RUT-C30 and QM9414 are both created by chemical and/or physical mutations but belong to different lineages [15]. It is conceivable that the genetic backgrounds of QM9414 and the two strains (QM6a and RUT-C30) are thus quite different. It is also well-known that cellular factors involved in DNA damage response [16], NHEJ, and HDR [17] affect the CRISPR/Cas9-mediated genome editing. Therefore, it is possible that the much different genetic backgrounds may account for the unexpected genome editing.

In contrast, direct transformation of the Cas9-gRNA complex into $T$. reesei TU-6, a uridine auxotrophic mutant of QM9414, led to genome editing in the predicted cbh1 locus. However, large DNA fragments including the co-transformed plasmid, chromosomal DNA, or their hybrid form, were found to be inserted in the targeted cbh1 gene. Insertion of large DNA fragments was 


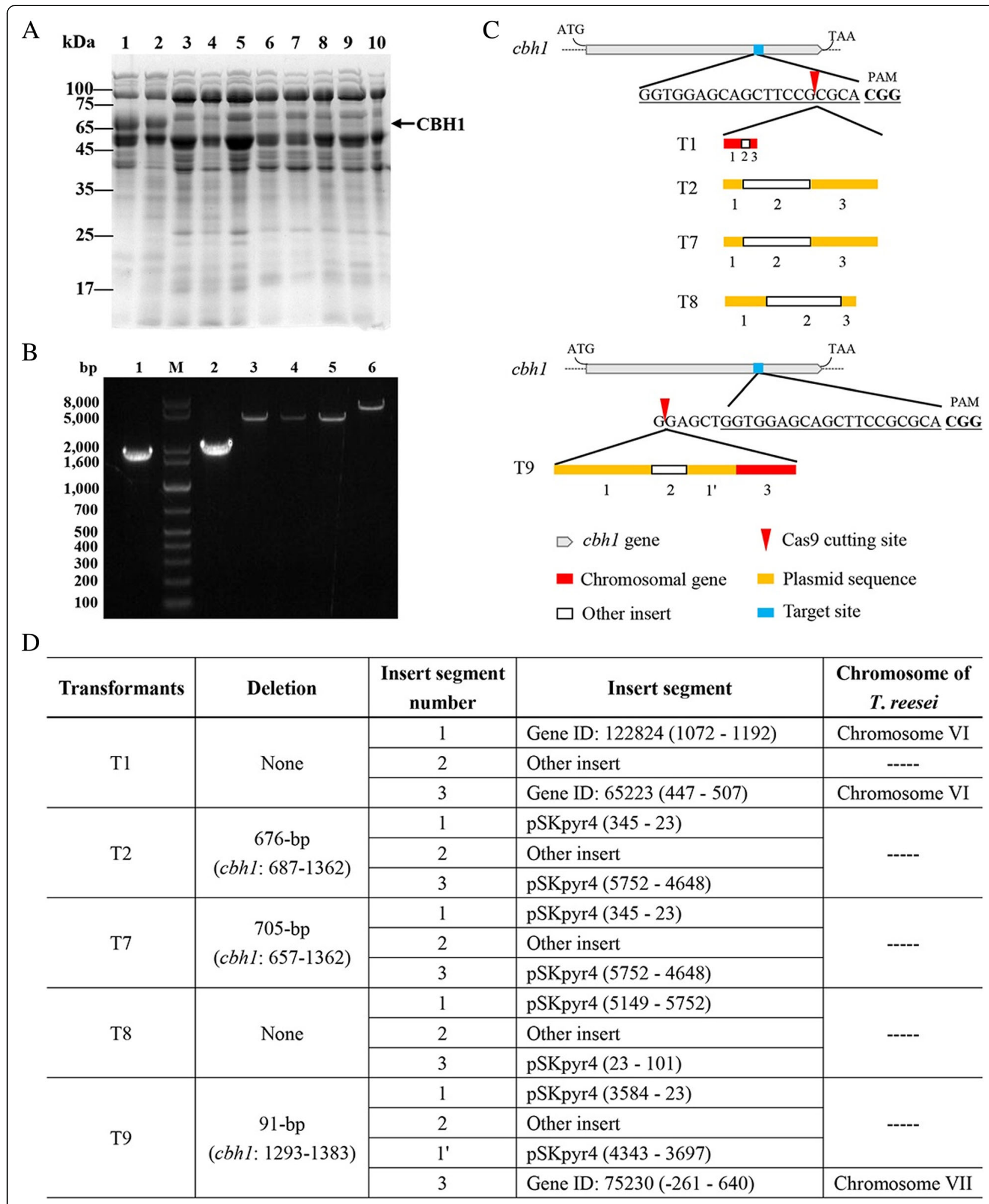

Fig. 3 (See legend on next page.) 
(See figure on previous page.)

Fig. 3 cbhl disruption in T. reesei by direct transformation of Cas9/gRNA complex and a plasmid containing the pyr4 selection marker. A: SDS-PAGE analysis of the fermentation supernatants of the transformants. M: protein molecular mass marker, lanes 1-2: TU-6; lanes 3-11: transformants (T1, T2, T3, T4, T6, T7, T8, and T9, respectively) that did not express $\mathrm{CBH}$ 1; B: agarose gel electrophoresis of the PCR products amplifying the cbh1 locus from the transformants that did not express CBH1. M: DNA molecular mass marker; lane 1: TU-6; lane 2-6: the transformants T1, T2, T7, T8, and T9, respectively; C: Schematic diagram showing the inserted DNA fragments in the edited cbh1 locus; D: Characteristics of inserted or deleted DNA fragments. The numbers for the T. reesei genes were counted from the start codon

not observed in Mucor circinelloide [9] and Fusarium oxysporum [11] when direct transformation of Cas9/ gRNA was employed. For these two fungi, only the Cas9/gRNA complex, but none exogenous DNA, was used in the transformation. In Nodulisporium sp., similar insertion of large DNA fragment was also observed. However, in that case an exogenous plasmid containing the selection marker was co-transformed with the in vitro transcribed gRNA into a recipient strain with in vivo expressed Cas9. Note, however, unlike the discovery reported herein, only the exogenous plasmid sequence was found in the inserted fragments in Nodulisporium sp. The promiscuity of the inserted fragments in T. reesei suggested complex DNA-repair events in the target locus.

With the success in cbh1 disruption, direct transformation of Cas9/gRNA complex provides a fast means for disruption of genes of interest in $T$. reesei. This method may also be used for HDR-mediated gene replacement. In a preliminary experiment we have observed gene replacement at the cel3c locus, albeit at a low frequency (5 out of 143 transformants), by co-transformation of Cas $9 /$ gRNA (targeting cel3c [18]) with a donor DNA in $T$. reesei (data not shown). Gene replacement efficiency can be improved by optimizing the amounts, nature and ratio of Cas9 and gRNA or using a strain with dysfunctional NHEJ DNA repair pathway.

\section{Conclusions}

The intracellularly expressed Cas9 led to biased off-target editing in ura5, which might be explained by the different strains used in our study and by other researchers. Direct transformation of Cas9/ gRNA complex allowed disruption of $c b h 1$ in $T$. reesei with large DNA inserts found in the edited locus. Compared with the previous report, our method avoids the time and labor needed for introducing the cas 9 gene into $T$. reesei and the uncertainty that may occur with its expression. In our hands, both in vivo expressed Cas9 and in vitro assembled Cas9/gRNA led to insertion of small or large DNA fragments during genome editing. This might be explained by the robust NHEJ process, which is the dominant DNA repair mechanism in $T$. reesei.

\section{Methods}

\section{Strains, plasmids and culture conditions}

The Escherichia coli Trans 1-T1 strain (Transgen, Beijing, China) was used as the host for plasmid construction and propagation throughout the study. The Saccharomyces cerevisiae AH109 strain (Clontech, San Francisco, CA) was used for construction of the cas9-expressing plasmid. The pPdc1-Cas9 plasmid for expressing the codon-optimized Streptococcus pyogenes cas 9 [7] was obtained by assembling cas 9 between the strong, constitutive $p d c 1$ promoter and $p d c 1$ terminator via DNA assembler using pRS424 (New England Biolabs, Beverly, MA) as a backbone plasmid [19]. The T. reesei QM9414 and its uridine auxotrophic strain TU-6 were maintained in our lab. T. reesei was grown in the minimal medium (MM, containing $\left(\mathrm{NH}_{4}\right)_{2} \mathrm{SO}_{4}, 5.0 \mathrm{~g} / \mathrm{L}$; $\mathrm{KH}_{2} \mathrm{PO}_{4}, 15 \mathrm{~g} / \mathrm{L} ; \mathrm{MgSO}_{4}, 0.6 \mathrm{~g} / \mathrm{L} ; \mathrm{CaCl}_{2}, 0.6 \mathrm{~g} / \mathrm{L} ; \mathrm{FeS}-$ $\mathrm{O}_{4} \cdot 7 \mathrm{H}_{2} \mathrm{O}, \quad 0.005 \mathrm{~g} / \mathrm{L} ; \quad \mathrm{MnSO}_{4} \cdot \mathrm{H}_{2} \mathrm{O}, \quad 0.0016 \mathrm{~g} / \mathrm{L}$; $\left.\mathrm{ZnSO}_{4} \cdot 7 \mathrm{H}_{2} \mathrm{O}, \quad 0.0014 \mathrm{~g} / \mathrm{L} ; \quad \mathrm{CoCl}_{2}, \quad 0.002 \mathrm{~g} / \mathrm{L}\right)$ supplemented with a certain kind of carbohydrate $(2 \%$ glucose for mycelial growth and $2 \%$ Avicel cellulose for cellulase induction) as the sole carbon source. For sporulation, $T$. reese $i$ was grown on potato dextrose agar (PDA) plates at $28^{\circ} \mathrm{C}$.

\section{Designing gRNA targeting chh1}

The gene cbh1 encoding the major cellulase cellobiohydrolase I (CBH1) was amplified from the genomic DNA of TU-6 by PCR using the primer pair Pcbh1F/ $\mathrm{R}$ (Additional file 1: Table S1). It was then ligated into pEASY-T3 (Transgen, Beijing, China) to obtain pT3cbh1. Three gRNA sequences for $c b h 1$ were designed using the online E-CRISPR design server (http://www.e-crisp.org/E-CRISP/). The corresponding encoding DNAs were synthesized in the form of single-stranded, complementary oligonucleotides (Additional file 1: Table S1). The T7 promoter (5'-TAATACGACTCACTATAGG-3') was designed upstream of the gRNAs. Equal amounts $(1 \mu \mathrm{g})$ of two complementary oligonucleotides were mixed, boiled for $10 \mathrm{~min}$, and cooled down at room temperature for annealing. Using the annealed double-stranded DNAs as the template, gRNAs were obtained by in vitro transcription with a MEGAshortscript T7 Transcription Kit (Invitrogen, Carlsbad, CA) and the RNAs 
were purified by using the RNA Purification Kit (Tiangen, Beijing, China). To determine the ability to guide digestion, $1 \mu \mathrm{g}$ of gRNA, $2 \mu \mathrm{g}$ of recombinant Cas9 (GenScript, Nanjing, China), and $200 \mathrm{ng}$ of the BcuI-linearized pT3cbh1 plasmid were mixed and incubated at $37^{\circ} \mathrm{C}$ for $2 \mathrm{~h}$. At the end of reaction, the samples were taken out for agarose gel electrophoresis analysis.

\section{Transformation of $T$. reesei}

For plasmid transformation, pPdc1-Cas9 and pRLMex30 (carrying the hygromycin B phosphotransferase gene) were co-introduced into the T. reesei QM9414 strain by polyethylene glycol (PEG)-mediated chemical transformation [20]. The transformants were selected on MM-glucose plates containing $150 \mu \mathrm{g} / \mathrm{ml}$ of hygromycin. For transformation of gRNA targeting ura5, $6.5 \mu \mathrm{g}$ of in vitro transcribed gRNA were similarly introduced into $T$. reesei through the same PEG-mediated protoplast transformation method. MM-glucose supplemented with 5-fluoroorotic acid (5-FOA, $3 \mathrm{mg} / \mathrm{ml}$ ) and $10 \mathrm{mM}$ uridine was used to select the transformants. Expression of wild-type, functional ura5 (encoding orotate phosploribosyl transferase) will convert 5-FOA into toxic 5'-fluorouridine monophosphate. For Cas9/gRNA transformation, $12 \mu \mathrm{g}$ of the Cas9 protein and $6.5 \mu \mathrm{g}$ of gRNA were mixed and incubated at $37^{\circ} \mathrm{C}$ for 30 min for in vitro assembly into the Cas9/gRNA RNP complex. The complex was then co-transformed with $6 \mu \mathrm{g}$ of the pSKpyr4 plasmid, which contains the pyr4 marker gene expression cassette [13], into the T. reesei TU-6 protoplasts.

\section{Analyzing cbh1 disruption in the transformants}

The spores $\left(1 \times 10^{8}\right)$ of TU-6 and its transformants were individually inoculated into $100 \mathrm{ml}$ of liquid MM-glucose (2\%) and cultured at $28^{\circ} \mathrm{C}$ with shaking for $48 \mathrm{~h}$. Mycelia were collected by filtering through a 200-mesh sifter, washed with MM, and transferred into the MM-Avicel cellulose (2\%) medium to induce cellulase expression. The fermentation broth on the eighth day after cellulose induction was analyzed by SDS-PAGE. The genomic DNAs of TU-6 and its transformants were extracted from the harvested mycelia and were used as templates for PCR amplification of the cbhl locus. The PCR conditions were $94^{\circ} \mathrm{C}$ for $1 \mathrm{~min}$ for initial denaturation, then 24 cycles including $94{ }^{\circ} \mathrm{C}$ for $25 \mathrm{~s}, 61{ }^{\circ} \mathrm{C}$ for $30 \mathrm{~s}$ (temperature reduced by $0.2^{\circ} \mathrm{C}$ per cycle), and $70^{\circ} \mathrm{C}$ for 5 min.

\section{Gene replacement of cel3c}

The Cas9 $(12 \mu \mathrm{g})$ was in vitro assembled with $6.5 \mu \mathrm{g}$ of gRNA targeting cel3c (GenBank accession number: AY281375.1) by incubation at $37^{\circ} \mathrm{C}$ for $30 \mathrm{~min}$. The donor DNA was consisted of two homology arms (1.0 kb each) flanking the cel3c locus. The homology arms were intercepted by an expression cassette for Melanocarpus albomyces laccase (GenBank accession number: CAE00180, under the control of $c b h 1$ promoter and terminator) and the pyr4 marker gene. The Cas9/gRNA complex and $3 \mu \mathrm{g}$ of the donor DNA were co-transformed into $T$. reesei protoplasts and the transformants were transferred into MM-lactose plate containing $0.4 \mathrm{mM}$ ABTS and $0.1 \mathrm{mM} \mathrm{CuSO}_{4}$. Laccase oxidizes ABTS and blue halos form around the laccase-expressing colonies. Laccase-positive transformants were grown on PDA plates for sporulation. Replacement of cel3c in these transformants was investigated using diagnostic PCR amplifying the fragments traversing across the chromosome and the cel3c editing locus.

\section{Additional file}

Additional file 1: Table S1. Primers used in this study. Figure S1. Sequencing the ura5 gene of QM9414, C5 expressing Cas9 intracellularly, and the C5-derived transformants. (DOCX $1220 \mathrm{~kb}$ )

\section{Abbreviations}

5-FOA: 5-fluoroorotic acid; CBH1: cellobiohydrolase I; CRISPR/Cas9: Clustered Regularly Interspaced Short Palindromic Repeats/CRISPR associated protein 9; HDR: homology-based DNA recombination; MM: minimal medium; NHEJ: non-homologous end-joining; PDA: potato dextrose agar; PEG: polyethylene glycol; RNP: ribonucleoprotein

\section{Acknowledgements}

We thank Prof. Lina Qin for scientific discussion.

\section{Funding}

This research was supported by the National Key R\&D Program of China (2016YFD0501409-02), the National Natural Science Foundation of China Grant 31672458, the Fundamental Research Funds for Central Non-profit Scientific Institution (1610382016004), and the Elite Youth Program of Chinese Academy of Agricultural Sciences.

Availability of data and materials

All data generated or analyzed during this study are included in this published article (and its supplementary information files)

\section{Authors' contributions}

XS designed the study; ZH carried out the experiments. Both authors analyzed the data, wrote the manuscript, and approved the final manuscript.

\section{Ethics approval}

Ethics approval was not needed for the study.

Consent for publication

Not applicable.

\section{Competing interests}

The authors declare that they have no competing interests.

\section{Publisher's Note}

Springer Nature remains neutral with regard to jurisdictional claims in published maps and institutional affiliations. 
Received: 30 September 2018 Accepted: 27 December 2018

Published online: 09 January 2019

References

1. Cherry JR, Fidantsef AL. Directed evolution of industrial enzymes: an update. Curr Opin Biotechnol. 2003;14(4):438-43.

2. IImen M, Saloheimo A, Onnela ML, Penttila ME. Regulation of cellulase gene expression in the filamentous fungus Trichoderma reesei. Appl Environ Microbiol. 1997;63(4):1298-306

3. Suominen PL, Mantyla AL, Karhunen T, Hakola S, Nevalainen H. High frequency one-step gene replacement in Trichoderma reesei. II. Effects of deletions of individual cellulase genes. Mol Gen Genet. 1993;241(5-6):523-30.

4. Seiboth B, Hakola S, Mach RL, Suominen PL, Kubicek CP. Role of four major cellulases in triggering of cellulase gene expression by cellulose in Trichoderma reesei. J Bacteriol. 1997;179(17):5318-20.

5. Landowski CP, Huuskonen A, Wahl R, Westerholm-Parvinen A, Kanerva A, Hanninen AL, Salovuori N, Penttila M, Natunen J, Ostermeier C, et al. Enabling low cost biopharmaceuticals: a systematic approach to delete proteases from a well-known protein production host Trichoderma reesei. PLoS One. 2015:10(8):e0134723.

6. Sternberg SH, Redding S, Jinek M, Greene EC, Doudna JA. DNA interrogation by the CRISPR RNA-guided endonuclease Cas9. Nature. 2014 507(7490):62-7.

7. Liu R, Chen L, Jiang YP, Zhou ZH, Zou G. Efficient genome editing in filamentous fungus Trichoderma reesei using the CRISPR/Cas9 system. Cell Discov. 2015:1.

8. Matsu-Ura T, Baek M, Kwon J, Hong C. Efficient gene editing in Neurospora crassa with CRISPR technology. Fungal Biol Biotechnol. 2015;2:4

9. Nagy G, Szebenyi C, Csernetics A, Vaz AG, Toth EJ, Vagvolgyi C, Papp T. Development of a plasmid free CRISPR-Cas9 system for the genetic modification of Mucor circinelloides. Sci Rep. 2017;7(1):16800.

10. Al Abdallah $Q$, Ge W, Fortwendel JR. A simple and universal system for gene manipulation in Aspergillus fumigatus: in vitro-assembled Cas9-guide RNA ribonucleoproteins coupled with microhomology repair templates. mSphere. 2017;2(6).

11. Wang Q, Cobine PA, Coleman JJ. Efficient genome editing in Fusarium oxysporum based on CRISPR/Cas9 ribonucleoprotein complexes. Fungal Genet Biol. 2018;117:21-9.

12. Su X, Schmitz G, Zhang M, Mackie Rl, Cann IK. Heterologous gene expression in filamentous fungi. Adv Appl Microbiol. 2012;81:1-61.

13. Qin LN, Cai FR, Dong XR, Huang ZB, Tao Y, Huang JZ, Dong ZY. Improved production of heterologous lipase in Trichoderma reesei by RNAi mediated gene silencing of an endogenic highly expressed gene. Bioresour Technol. 2012;109:116-22.

14. Zheng YM, Lin FL, Gao H, Zou G, Zhang JW, Wang GQ, Chen GD, Zhou ZH, Yao XS, Hu D. Development of a versatile and conventional technique for gene disruption in filamentous fungi based on CRISPR-Cas9 technology. Sci Rep. 2017:7(1):9250.

15. Seidl V, Gamauf C, Druzhinina IS, Seiboth B, Hartl L, Kubicek CP. The Hypocrea jecorina (Trichoderma reesei) hypercellulolytic mutant RUT C30 lacks a $85 \mathrm{~kb}$ (29 gene-encoding) region of the wild-type genome. BMC Genomics. 2008;9:327.

16. Ihry RJ, Worringer KA, Salick MR, Frias E, Ho D, Theriault K, Kommineni S, Chen J, Sondey M, Ye C, et al. p53 inhibits CRISPR-Cas9 engineering in human pluripotent stem cells. Nat Med. 2018:24(7):939-46.

17. Chu VT, Weber T, Wefers B, Wurst W, Sander S, Rajewsky K, Kuhn R. Increasing the efficiency of homology-directed repair for CRISPR-Cas9induced precise gene editing in mammalian cells. Nat Biotechnol. 2015; 33(5):543-8.

18. Qin L, Jiang X, Dong Z, Huang J, Chen X. Identification of two integration sites in favor of transgene expression in Trichoderma reesei. Biotechnol Biofuels. 2018;11:142.

19. Shao ZY, Zhao H, Zhao HM. DNA assembler, an in vivo genetic method for rapid construction of biochemical pathways. Nucleic Acids Res. 2009:37(2).

20. Penttila M, Nevalainen $H$, Ratto M, Salminen E, Knowles J. A versatile transformation system for the cellulolytic filamentous fungus Trichoderma reesei. Gene. 1987;61(2):155-64.

Ready to submit your research? Choose BMC and benefit from:

- fast, convenient online submission

- thorough peer review by experienced researchers in your field

- rapid publication on acceptance

- support for research data, including large and complex data types

- gold Open Access which fosters wider collaboration and increased citations

- maximum visibility for your research: over $100 \mathrm{M}$ website views per year

At BMC, research is always in progress.

Learn more biomedcentral.com/submissions 